ALEA, Lat. Am. J. Probab. Math. Stat. 17, 877-900 (2020)

DOI: 10.30757/ALEA.v17-34

\title{
Critical branching processes in random environment and Cauchy domain of attraction
}

\section{Dong, C. Smadi* and V. A. Vatutin}

Xidian University

266 Xinglong Section of Xifeng Road,

Xi'an, Shaanxi, 710126, China.

E-mail address: czdong@xidian.edu.cn

Univ. Grenoble Alpes, INRAE, LESSEM, and Univ. Grenoble Alpes, CNRS, Institut Fourier

2 rue de la papeterie,

38402 Saint-Martin d'Hères, France.

E-mail address: charline.smadi@inrae.fr

URL: https://www-fourier . univ-grenoble-alpes.fr/ smadic/

Department of Discrete Mathematics, Steklov Mathematical Institute of Russian Academy of Sciences

8 Gubkin Street,

117966 Moscow GSP-1, Russia.

E-mail address: vatutin@mi.ras.ru

URL: http://www.mathnet.ru/eng/person20403

\begin{abstract}
We are interested in the survival probability of a population modeled by a critical branching process in an i.i.d. random environment and in the growth rate of the population given its survival up to a large time $n$. We assume that the random walk associated with the branching process is oscillating and satisfies a Doney-Spitzer condition $\mathbf{P}\left(S_{n}>0\right) \rightarrow \rho, n \rightarrow \infty$, which is a standard condition in fluctuation theory of random walks. Unlike the previously studied case $\rho \in(0,1)$, we investigate the case where the offspring distribution is in the domain of attraction of an asymmetric stable law with parameter 1 , which implies that $\rho=0$ or 1 . We find the asymptotic behaviour of the survival probability of the population and prove a Yaglom-type conditional limit theorem for the population size in these two cases.
\end{abstract}

Received by the editors November 5th, 2019; accepted August 13th, 2020.

2010 Mathematics Subject Classification. 60J80, 60G50.

Key words and phrases. Branching process, random environment, random walk, conditioned random walk, Spitzer's condition.

${ }^{*}$ Corresponding author. 


\section{Introduction and main results}

Branching processes have been introduced independently by Bienaymé (1845) and by Watson and Galton (1875) in the 19th century in order to study the extinction of family names. Since then they have been widely used to model the dynamics of populations or the spread of infections for instance Haccou et al. (2007); Allen (2011). Branching processes in random environment have been first introduced and studied by Smith and Wilkinson and Athreya and Karlin in the early seventies Smith and Wilkinson (1969); Athreya and Karlin (1971b,a). By introducing such processes, their aim was to better understand the effect of the environmental stochasticity on the population dynamics. Initially restricted to environments satisfying strong assumptions or to particular offspring distributions, they have been later generalised. Their study has known a renewed interest during the last two decades, with the development of new techniques to investigate them, in particular by linking events on the trajectory of the population process until a certain generation $n$ with an other event of its associated random walk until the same time $n$ (see, for instance, Afanasyev et al., 2005, 2012; Bansaye and Böinghoff, 2013; Vatutin et al., 2013 for more detail).

A branching process in an independent identically distributed (i.i.d.) random environment is specified by a sequence of i.i.d. random offspring generating functions

$$
f_{n}(s):=\sum_{k=0}^{\infty} f_{n}[k] s^{k}, \quad n \in\{1,2, \ldots\}=: \mathbb{N}, \quad 0 \leq s \leq 1 .
$$

Denoting by $Z_{n}$ the number of individuals in the process at time $n$, we assume that there is initially one individual in the population $\left(Z_{0}=1\right)$ and we define the population size evolution by the relations

$$
\mathbf{E}\left[s^{Z_{n}} \mid f_{1}, \ldots, f_{n} ; Z_{0}, Z_{1}, \ldots, Z_{n-1}\right]:=\left(f_{n}(s)\right)^{Z_{n-1}}, \quad n \in \mathbb{N} .
$$

Let

and denote

$$
X_{k}:=\log f_{k}^{\prime}(1)=\log \mathbf{E}\left[Z_{k} \mid f_{k}, Z_{k-1}=1\right], \quad k \in \mathbb{N}
$$

$$
S_{0}:=0, \quad S_{n}:=X_{1}+X_{2}+\ldots+X_{n}
$$

the auxiliary random walk associated with the quenched expectation of offspring number. The long time behaviour of the process $\mathcal{Z}:=\left\{Z_{n}, n \geq 0\right\}$ is intimately related to the properties of the random walk $\mathcal{S}:=\left\{S_{n}, n \geq 0\right\}$ (see Geiger and Kersting (2000); Geiger et al. (2003); Afanasyev et al. (2005) for instance). According to fluctuation theory of random walks (see Feller, 1950), three different cases are possible: either $\mathcal{S}$ drifts to $\infty$, or $\mathcal{S}$ drifts to $-\infty$, or the random walk oscillates:

$$
\limsup _{n \rightarrow \infty} S_{n}=+\infty \text { and } \liminf _{n \rightarrow \infty} S_{n}=-\infty
$$

with probability 1 . Accordingly, the branching process is called supercritical, subcritical, or critical Afanasyev et al. (2005). We consider the last possibility. In this case the stopping time

$$
T^{-}:=\min \left\{k \geq 1: S_{k}<0\right\}
$$

is finite with probability 1 and, as a result (see Afanasyev et al., 2005), the extinction time

$$
\mathfrak{T}:=\min \left\{k \geq 1: Z_{k}=0\right\}
$$

of the process $\mathcal{Z}$ is finite with probability 1 . 
In this work we will be interested in the asymptotic behaviour of the survival probability $\mathbf{P}\left(Z_{n}>0\right)$ of the population at large time and in the growth rate of the process $\left\{Z_{k}, 0 \leq k \leq n\right\}$ given $Z_{n}>0$. They are natural problems when dealing with populations, and they have been answered under various assumptions in the case of branching processes in random environment (see, for instance, Kozlov (1976); Geiger and Kersting (2000); Dyakonova et al. (2004); Afanasyev et al. (2005, 2012); Vatutin and Dyakonova (2017); Vatutin and D'yakonova (2019)).

We assume that the random walk $\mathcal{S}$ satisfies the Doney-Spitzer condition, which is a classical condition in fluctuation theory, and writes

$$
\lim _{n \rightarrow \infty} \frac{1}{n} \sum_{m=1}^{n} \mathbf{P}\left(S_{m}>0\right)=: \rho .
$$

According to Bertoin and Doney (1997), this condition is equivalent to

$$
\lim _{n \rightarrow \infty} \mathbf{P}\left(S_{n}>0\right)=: \rho \text {. }
$$

The case $\rho \in(0,1)$ has been studied by Afanasyev and his coauthors in Afanasyev et al. (2005). Under some mild additional assumptions they proved the following equivalent for the survival probability of the population at large times $n$,

$$
\mathbf{P}\left(Z_{n}>0\right) \sim \frac{l(n)}{n^{1-\rho}},
$$

where $l(\cdot)$ is a slowly varying function. They also proved that, as $n \geq r_{n} \rightarrow \infty$

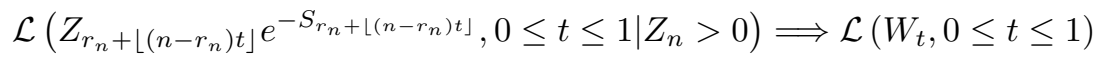

where $W_{t}=W$ for all $0 \leq t \leq 1$ and $W$ is a random variable meeting the condition $\mathbf{P}(0<W<\infty)=1$.

Here the symbol $\Longrightarrow$ stands for weak convergence with respect to the Skorokhod topology in the space $D[0,1]$ of càdlàg functions on the unit interval.

The aim of the present paper is to complement (1.2) and (1.3) by considering as $n \rightarrow \infty$ the asymptotic behaviour of $\mathbf{P}\left(Z_{n}>0\right)$ and proving a Yaglom-type limit theorem for the scaled population size of $\left\{Z_{k}, 0 \leq k \leq n\right\}$ given $Z_{n}>0$ in the cases $\rho=0$ and $\rho=1$.

Before stating our main results, we need to introduce some notation and a set of assumptions on the law of the random walk $\mathcal{S}$. The main assumption is that $\mathcal{S}$ is in the domain of attraction of a stable law with parameter 1 . It means that there exist a slowly varying function $\mathfrak{l}(\cdot)$, and two nonnegative numbers $p$ and $q, p+q=1$, such that

$$
\mathbf{P}\left(X_{1}>x\right) \sim p \frac{\mathfrak{l}(x)}{x} \text { and } \mathbf{P}\left(X_{1}<-x\right) \sim q \frac{\mathfrak{l}(x)}{x}, \quad x \rightarrow \infty .
$$

As we will see (Remark 1.1), $\mathcal{S}$ will satisfy the Doney-Spitzer condition with $\rho=0$ (resp. $\rho=1$ ) in the case $p>q$ (resp. $p<q$ ). To show that we introduce two scaling sequences which play the main role in the asymptotic behaviour of various quantities related to the random walk $\mathcal{S}$. The first sequence, $\left\{a_{n}, n \geq 1\right\}$, satisfies, as $n \rightarrow \infty$ the relation

$$
\frac{\mathfrak{l}\left(a_{n}\right)}{a_{n}} \sim \frac{1}{n}
$$


Note that the sequence is regularly varying with parameter 1 as $n \rightarrow \infty$ (see Seneta, 1976). We can thus rewrite it as

$$
a_{n}=n \mathfrak{l}_{1}(n)
$$

where $\mathfrak{l}_{1}($.$) is a slowly varying function as n \rightarrow \infty$. The second sequence, $\left\{h_{n}, n \in \mathbb{N}\right\}$, is specified by

$$
h_{n}:=n \mu\left(a_{n}\right) \text { with } \mu(x)=\mathbf{E}\left[X_{1} \mathbf{1}_{\left\{\left|X_{1}\right| \leq x\right\}}\right],
$$

where $\mathbf{1}_{\{A\}}$ is the indicator of the event $A$.

In addition, we suppose that the random walk $S$ is oscillating, that is to say,

$$
\mu:=\mathbf{E}\left[X_{1}\right]=0 .
$$

Let

$$
\mathfrak{l}^{*}(z):=\int_{z}^{\infty} \frac{\mathfrak{l}(y)}{y} d y .
$$

Let us make some obsevations on $p, q$ and $\rho$ before going further.

Remark 1.1. Assume that conditions (1.4) and (1.8) hold. Then according to Theorem 2.1, $h_{n} \rightarrow-\infty$ (resp. $+\infty$ ) when $p>q$ (resp. $p<q$ ). Moreover, from Rogozin (1976), $S_{n} / h_{n}$ converges to 1 in probability when $n$ goes to infinity (indeed when $q>p, \mu$ is positive and slowly varying at infinity, and $x \mathbf{P}\left(\left|X_{1}\right|>x\right) / \mu(x) \sim$ $\mathfrak{l}(x) /\left((q-p) \mathfrak{l}^{*}(x)\right) \rightarrow 0$ at infinity, and symmetric conditions in the case $\left.q<p\right)$. We deduce that $S_{n} \rightarrow-\infty$ if $p>q$ (hence $\rho=0$ ) and $S_{n} \rightarrow+\infty$ if $p<q$ (hence $\rho=1)$.

Remark 1.2. In the case $p=q, \rho$ may take different values depending on the asymptotics of $h_{n} / a_{n}$ for large $n$. More precisely, we may have at least the following three cases:

- If $h_{n} / a_{n} \rightarrow b \in \mathbb{R}$ when $n \rightarrow \infty$,

$$
\rho=\frac{1}{2}+\frac{1}{\pi} \arctan \left(\frac{2 b}{\pi}\right) \in(0,1)
$$

(see (iii) p.35 in Berger (2019)).

- If $h_{n} / a_{n} \rightarrow \infty$ when $n \rightarrow \infty$, then $\rho=1$ (see Equation (7.20) in Berger, 2019).

- Symmetrically, if $h_{n} / a_{n} \rightarrow-\infty$ when $n \rightarrow \infty$, then $\rho=0$.

Remark 1.3. There are more exotic cases than (1.4) where $\rho=0$ or $\rho=1$ under condition (1.8). We focus on processes satisfying (1.4), as in this case the symmetry makes our results simpler to express.

As in Afanasyev et al. (2005), we need to impose restrictions on the standardized truncated second moment of the environment, namely:

$$
\zeta_{k}(a):=\sum_{y=a}^{\infty} y^{2} f_{k}[y] /\left(\sum_{y=0}^{\infty} y f_{k}[y]\right)^{2},
$$

for $a, k \in \mathbb{N}$. The moment condition depends on the value of $\rho$ in the Doney-Spitzer condition (1.1).

Condition A. $(\rho=0 \leftrightarrow p>q)$ There exist $a \in \mathbb{N}$ and $\beta>0$ such that

$$
\mathbf{E}\left[\zeta_{1}^{\beta}(a)\right]<\infty \text { and } \mathbf{E}\left[U\left(X_{1}\right) \zeta_{1}^{\beta}(a)\right]<\infty,
$$


where $U$ is the renewal function associated with the strict descending ladder epochs of $\mathcal{S}$,

$$
\gamma_{0}:=0, \quad \gamma_{j+1}:=\min \left(n>\gamma_{j}: S_{n}<S_{\gamma_{j}}\right), \quad j \in \mathbb{N}_{0}:=\mathbb{N} \cup\{0\},
$$

and is defined by

$$
U(x):=\sum_{j=0}^{\infty} \mathbf{P}\left(S_{\gamma_{j}} \geq-x\right), \quad x>0, \quad U(0)=1, \quad U(x)=0, \quad x<0 .
$$

Condition B. $(\rho=1 \leftrightarrow p<q)$ There exist $a \in \mathbb{N}$ and $\beta>0$ such that

$$
\mathbf{E}\left[\left(\log ^{+} \zeta_{1}(a)\right)^{1+\beta}\right]<\infty \text { and } \mathbf{E}\left[U\left(X_{1}\right)\left(\log ^{+} \zeta_{1}(a)\right)^{1+\beta}\right]<\infty \text {. }
$$

Recall that the moment condition in Afanasyev et al. (2005) under the DoneySpitzer condition (1.1) with $\rho \in(0,1)$ was the existence of $a \in \mathbb{N}$ and $\beta>0$ such that:

$$
\mathbf{E}\left[\left(\log ^{+} \zeta_{1}(a)\right)^{1 / \rho+\beta}\right]<\infty \text { and } \mathbf{E}\left[U\left(X_{1}\right)\left(\log ^{+} \zeta_{1}(a)\right)^{1+\beta}\right]<\infty .
$$

Our Condition B is thus a natural extension of the moment condition to the case $\rho=1$. In contrast, such a natural extension for $\rho=0$ would have provided an infinite exponent for the logarithm and we could not obtain a moment condition on the logarithm only. Notice however that we can take $\beta$ as small as we want in Condition A. Thus, our moment condition is not very strong.

Last, for technical reasons, we need to add an assumption which will be used for the case $p>q$ only.

Condition C. There exists a function $g(x)=e^{o(x)}, x \rightarrow \infty$, such that

$$
\sum_{j=1}^{\infty} 1 / \Lambda(g(j))<\infty
$$

where $\Lambda$ is a slowly varying function (see the proof of Proposition 12 in Kortchemski and Richier, 2019), defined by

$$
\Lambda\left(\frac{1}{1-s}\right):=\exp \left(\sum_{k=1}^{\infty} \frac{\mathbf{P}\left(S_{k} \geq 0\right)}{k} s^{k}\right), \quad s \in[0,1) .
$$

This assumption is needed to ensure that the population has a positive probability to survive in a 'good' environment, that is to say in an environment conditioned to stay positive (see Lemma 4.1).

We give now examples of branching processes in random environment satisfying Conditions A and B. Some of them also meet Conditions $\mathbf{C}$, and some of them do not, as we will show.

Since $U(x), x \geq 0$, is a renewal function, there exists a constant $C \in(0, \infty)$ such that $U(x) \leq C\left(1+x^{+}\right)$. Given condition (1.8) it follows that $\mathbf{E}\left[U\left(X_{1}\right)\right]<\infty$. This observation shows that Conditions $\mathbf{A}$ and $\mathbf{B}$ hold true if $\zeta_{1}(a)$ is bounded from above for some $a$. 
Example 1.4. Assume that the offspring generating functions are geometric with probability 1:

$$
f_{n}(s)=\frac{1-b_{n}}{1-s b_{n}}
$$

where $0<b_{n}<1$ are random and i.i.d. and such that

$$
X_{n}=\log f_{n}^{\prime}(1)=\log \frac{b_{n}}{1-b_{n}}, \quad n \in \mathbb{Z}_{+}
$$

satisfy (1.4). We can check that if condition (1.14) is valid then $\zeta_{1}(2) \leq 4$ (see, for instance, Example 5.2, page 103 in Kersting and Vatutin, 2017). Thus, Conditions $\mathbf{A}$ and $\mathbf{B}$ are satisfied.

We will now focus on the case $p>q$ and give two examples of sequences $\left(b_{n}, n \in \mathbb{N}\right)$ such that (1.14) holds: the first process meets Condition $\mathbf{C}$, the second one does not.

- Assume first that

$$
\mathbf{P}\left(b_{1} \in[1-y, 1-y+d y]\right) \sim \frac{p d y}{y \ln ^{2} y(\ln \ln 1 / y)^{2}}, \quad(y \rightarrow 0) .
$$

Then we get that $\mathfrak{l}(x)=1 / \ln ^{2} x, \mathfrak{l}^{*}(x)=1 / \ln x$, and $a_{n} \sim n / \ln ^{2} n$ for large $n$. From Equation (7.23) in Berger (2019), we know that

$$
\sum_{k=1}^{\infty} \frac{\mathbf{P}\left(S_{k} \geq 0\right)}{k} s^{k} \sim-\frac{p}{p-q} \ln \mathfrak{l}^{*}\left(\mu_{1 /(1-s)}\right), \quad(s \rightarrow 1),
$$

and we also have (see p. 13 in Berger (2019)) that

$$
\mathfrak{l}^{*}\left(\mu_{n}\right) \sim \mathfrak{l}^{*}\left(a_{n}\right), \quad(n \rightarrow \infty) .
$$

We deduce from these two asymptotics that when $s$ is close to 1 ,

$$
\begin{aligned}
\sum_{k=1}^{\infty} \frac{\mathbf{P}\left(S_{k} \geq 0\right)}{k} s^{k} & \sim-\frac{p}{p-q} \ln \mathfrak{l}^{*}\left(a_{1 /(1-s)}\right) \\
& \sim-\frac{p}{p-q} \ln \mathfrak{l}^{*}\left(1 /\left((1-s) \ln ^{2}(1-s)\right)\right) \\
& \sim \frac{p}{p-q} \ln \ln (1 /(1-s))=\ln \left((-\ln (1-s))^{p /(p-q)}\right) .
\end{aligned}
$$

Hence

$$
\begin{aligned}
\ln \Lambda\left(\frac{1}{1-s}\right) & =\sum_{k=1}^{\infty} \frac{\mathbf{P}\left(S_{k} \geq 0\right)}{k} s^{k} \\
& =\ln \left((-\ln (1-s))^{p /(p-q)}\right)+\varepsilon(1-s) \ln \ln (1 /(1-s))
\end{aligned}
$$

with $\varepsilon(y)$ converging to 0 when $y$ goes to 0 . Now, if we replace $s$ by $1-e^{-j / \ln j}$ which goes to 1 when $j$ goes to infinity, we get for $j$ large enough

$$
\Lambda\left(e^{j / \ln j}\right)=\left(\frac{j}{\ln j}\right)^{p /(p-q)+\varepsilon\left(e^{-j / \ln j}\right)} \geq\left(\frac{j}{\ln j}\right)^{(p+1) / 2 p},
$$

where we used that the function $p \mapsto p /(2 p-1)$ is decreasing on $(1 / 2,1)$ and that $\varepsilon\left(e^{-j / \ln j}\right)$ goes to 0 as $j$ goes to $\infty$. Condition $\mathbf{C}$ is thus satisfied. 
- Now assume that

$$
\mathbf{P}\left(b_{1} \in[1-y, 1-y+d y]\right) \sim \frac{p d y}{y(\ln \ln y)(\ln \ln \ln 1 / y)^{2}}, \quad(y \rightarrow 0) .
$$

Then $\mathfrak{l}(x)=1 /\left(\ln x(\ln \ln x)^{2}\right), \mathfrak{l}^{*}(x)=1 / \ln \ln x$, and $a_{n} \sim n /\left(\ln n(\ln \ln n)^{2}\right)$ for large $n$. Similar calculations as in for the previous examples yield that

$$
\Lambda(x)=(\ln \ln x)^{p /(p-q)+\varepsilon(1 / x)},
$$

with $\varepsilon($.$) going to 0$ at 0 . In particular, if we take a function $f$ such that for large $j, f(j) \leq e^{j}$, we obtain for $j$ large enough

$$
\Lambda(f(j)) \leq(\ln j)^{2 p /(p-q)}=o(j) .
$$

We deduce that Condition $\mathbf{C}$ is not satisfied for this example.

Example 1.5. Assume that the offspring generating functions are Poisson with probability 1:

$$
f_{n}(s)=e^{\lambda_{n}(s-1)}
$$

where $\lambda_{n}, n \in \mathbb{Z}_{+}$are i.i.d. and $X_{1}=\log \lambda_{1}$ satisfies (1.4). Direct calculations show that $\zeta_{1}(2) \leq 2$ (see Example 5.2., page 103 in Kersting and Vatutin, 2017). We can provide examples satisfying or not Condition $\mathbf{C}$ similarly as the previous case.

As previously observed under different assumptions on the random environment (see, for instance, Vatutin et al., 2013 for a comprehensive review on the critical and subcritical cases (before 2013) or the recent monograph Kersting and Vatutin, 2017) the survival of a branching process in random environment is essentially determined by its survival until the moment when the associated random walk $\mathcal{S}$ attains its infimum. The idea is that if we divide the trajectory of the process on the interval $[0, n]$ into two parts, one before the running infimum of the random environment $\mathcal{S}$, and one after this running infimum, the process will live in a favorable environment after the running infimum of the random environment, and will thus survive with a nonnegligible probability until time $n$, provided it survived until the time of the running infimum. This is essentially, in words, the idea of the proof of our main result (see Theorem 1.6). To state things more rigorously, we introduce the running infimum of the random walk $\mathcal{S}$ :

$$
L_{n}:=\min \left\{S_{0}, S_{1}, \ldots, S_{n}\right\}, n \in \mathbb{N}_{0} .
$$

Depending on the relative positions of $p$ and $q$ (defined in (1.4)) or equivalently on the value of $\rho$ ( 0 or 1$)$ we have the two following possible asymptotics for the survival probability of the process $\mathcal{Z}$ :

Theorem 1.6. Assume that Conditions (1.4) and (1.8) hold.

- If $p>q$, and Conditions $\boldsymbol{A}$ and $\boldsymbol{C}$ hold then there exists a constant $K_{1} \in(0, \infty)$ such that, as $n \rightarrow \infty$

$$
\mathbf{P}\left(Z_{n}>0\right) \sim K_{1} \mathbf{P}\left(L_{n} \geq 0\right) \sim K_{1} \frac{\mathfrak{l}_{2}(n)}{n},
$$

where $\mathfrak{l}_{2}($.$) is a function slowly varying at infinity.$

- If $p<q$ and Condition $\boldsymbol{B}$ holds then there exists a constant $K_{2} \in(0, \infty)$ such that, as $n \rightarrow \infty$

$$
\mathbf{P}\left(Z_{n}>0\right) \sim K_{2} \mathbf{P}\left(L_{n} \geq 0\right) \sim K_{2} \mathfrak{l}_{3}(n),
$$

where $\mathfrak{l}_{3}($.$) is a function slowly varying at infinity.$ 
Hence, despite the irregular behaviour of the associated random walk $\mathcal{S}$ (a null expectation but a probability converging to 1 to be positive (resp. negative)), the asymptotic behaviour of the survival probability is, except for the slowly varying function, the limit of the one obtained in Afanasyev et al. (2005) by taking $\rho=0$ or 1 instead of $\rho \in(0,1)$.

Now we investigate the behaviour of the process $\left\{Z_{k}, 0 \leq k \leq n\right\}$ given $\left\{Z_{n}>0\right\}$. To this aim, let us introduce the process $X^{r, n}=\left\{X_{t}^{r, n}, 0 \leq t \leq 1\right\}$ where

$$
X_{t}^{r, n}:=Z_{r+\lfloor(n-r) t\rfloor} e^{-S_{r+\lfloor(n-r) t\rfloor},} \quad 0 \leq t \leq 1 .
$$

The following Yaglom-type theorem is an analogue of Theorem 1.3 in Afanasyev et al. (2005).

Theorem 1.7. Assume that Conditions (1.4) and (1.8) hold. Let $\left(r_{n}, n \in \mathbb{Z}_{+}\right)$be a sequence of positive integers such that $r_{n} \leq n$ and $r_{n} \rightarrow \infty$ as $n \rightarrow \infty$. If $p>q$ and Conditions $\boldsymbol{A}$ and $\boldsymbol{C}$ hold or $p<q$ and Condition $\boldsymbol{B}$ holds then

$$
\mathcal{L}\left(X^{r, n} \mid Z_{n}>0\right) \Longrightarrow \mathcal{L}\left(W_{t}, 0 \leq t \leq 1\right), \quad(n \rightarrow \infty),
$$

where

$$
\mathbf{P}\left(W_{t}=W \text { for all } t \in[0,1]\right)=1
$$

for a random variable $W$ such that $\mathbf{P}(0<W<\infty)=1$.

We see that similarly to the case $\rho \in(0,1)$ the growth of $\mathcal{Z}$ is mainly specified by the properties of the random walk $\mathcal{S}$.

The rest of the paper is structured as follows. Section 2 is dedicated to the study of the running extrema of the random walk $S$. In Section 3, we perform a change of measure, obtained as a Doob-h transform, where the renewal function $U(\cdot)$ of $\mathcal{S}$ and the indicator of the event $\left\{L_{n} \geq 0\right\}$ are involved. Finally, the proofs of the main results, Theorems 1.6 and 1.7 are completed in Sections 4 and 5.

\section{Estimates for the suprema of the associated random walk}

The aim of this section is to provide some bounds for the probabilities of the events related to the running infimum and maximum of the random walk $\mathcal{S}$. We recall the definition of the running infimum in (1.15), and introduce the running maximum via

$$
M_{n}:=\max \left\{S_{1}, \ldots, S_{n}\right\}, n \in \mathbb{N} .
$$

We first list a number of known results which will be needed in our arguments. Recall definitions (1.5) and (1.7). The following results have been first derived in Theorem 3.4 in Berger (2019), and then under weaker conditions in Kortchemski and Richier (2019) (see Proposition 12 and Remark 13).

Theorem 2.1. Assume that Conditions (1.4) and (1.8) hold. Then when $n$ goes to infinity,

1) if $p>q$ then $h_{n} \sim-(p-q) n \mathfrak{l}^{*}\left(a_{n}\right) \rightarrow-\infty$ and

$$
\mathbf{P}\left(L_{n} \geq 0\right) \sim \frac{\mathfrak{l}\left(\left|h_{n}\right|\right)}{\left|h_{n}\right|} \Lambda(n)=: \frac{\mathfrak{l}_{2}(n)}{n},
$$

for some slowly varying functions $\mathfrak{l}_{2}$ (recall that the sowly varying function $\Lambda$ has been defined in (1.13)). 
2) if $p<q$ then $h_{n} \sim(q-p) n \mathfrak{l}^{*}\left(a_{n}\right) \rightarrow+\infty$ and

$$
\mathbf{P}\left(L_{n} \geq 0\right) \sim \frac{1}{\tilde{\Lambda}(n)}=: \mathfrak{l}_{3}(n),
$$

for some slowly varying functions $\mathfrak{l}_{3}$. The slowly varying function $\tilde{\Lambda}$ is defined as $\Lambda$ (recall (1.13)) but with $-\mathcal{S}$ in place of $\mathcal{S}$, and with strict inequalities (i.e. $\mathbf{P}\left(-S_{k}>0\right)$ in the sum $)$.

3) if $p>q$ then

$$
\mathbf{P}\left(M_{n}<0\right) \sim \frac{1}{\Lambda(n)}=: \mathfrak{l}_{4}(n)
$$

4) if $p<q$ then

$$
\mathbf{P}\left(M_{n}<0\right) \sim \frac{\mathfrak{l}\left(\left|h_{n}\right|\right)}{\left|h_{n}\right|} \tilde{\Lambda}(n)=: \frac{\mathfrak{l}_{5}(n)}{n} .
$$

Recall the definitions of the strict descending ladder epochs $\left\{\gamma_{j}, j \in \mathbb{N}_{0}\right\}$ of $\mathcal{S}$ and of their associated renewal function $U(\cdot)$ in (1.11) and (1.12), respectively, and introduce the strict ascending ladder epochs $\left\{\Gamma_{j}, j \in \mathbb{N}_{0}\right\}$ of $\mathcal{S}$ and their associated renewal function $V(\cdot)$ via

$$
\Gamma_{0}:=0, \quad \Gamma_{j+1}:=\min \left(n>\Gamma_{j}: S_{n}>S_{\Gamma_{j}}\right), \quad j \in \mathbb{N}_{0},
$$

and

$$
V(x):=1+\sum_{j=1}^{\infty} \mathbf{P}\left(S_{\Gamma_{j}}<x\right), \quad x>0, \quad V(0)=1, \quad V(x)=0, \quad x<0 .
$$

For a slowly varying function $\mathfrak{l}_{i}(\cdot)$ let

$$
\hat{\mathfrak{l}}_{i}(n):=\int_{1}^{n} \frac{\mathfrak{l}_{i}(x)}{x} d x .
$$

The next lemma provides bounds on the probabilities for the running extrema to be in a certain interval.

Lemma 2.2. Assume that Conditions (1.4) and (1.8) hold. Then there exists a constant $C \in(0, \infty)$ such that, for every $x \geq 0$ and $n \in \mathbb{N}$,

$$
\mathbf{P}\left(L_{n} \geq-x\right) \leq\left\{\begin{array}{c}
C U(x) n^{-1} \hat{\mathfrak{l}}_{2}(n) \quad \text { if } p>q, \\
C U(x) \mathfrak{l}_{3}(n) \quad \text { if } p<q,
\end{array}\right.
$$

and

$$
\mathbf{P}\left(M_{n}<x\right) \leq\left\{\begin{array}{c}
C V(x) \mathfrak{l}_{4}(n) \quad \text { if } p>q, \\
C V(x) n^{-1} \hat{\mathfrak{l}}_{5}(n) \quad \text { if } \quad p<q .
\end{array}\right.
$$

Proof: We know by a Spitzer identity that, for any $\lambda \geq 0$

$$
\begin{aligned}
\sum_{n=0}^{\infty} s^{n} \mathbf{E}\left[e^{\lambda L_{n}}\right] & =\exp \left\{\sum_{n=1}^{\infty} \frac{s^{n}}{n} \mathbf{E}\left[e^{\lambda \min \left(0, S_{n}\right)}\right]\right\} \\
& =\exp \left\{\sum_{n=1}^{\infty} \frac{s^{n}}{n} \mathbf{E}\left[e^{\lambda S_{n}} ; S_{n}<0\right]\right\} \exp \left\{\sum_{n=1}^{\infty} \frac{s^{n}}{n} \mathbf{P}\left(S_{n} \geq 0\right)\right\} .
\end{aligned}
$$


A Sparre-Anderson identity (see, for instance, Theorem 4.3 in Kersting and Vatutin (2017)) allows us to rewrite the first term at the right hand side as

$$
\begin{aligned}
\exp \left\{\sum_{n=1}^{\infty} \frac{s^{n}}{n} \mathbf{E}\left[e^{\lambda S_{n}} ; S_{n}<0\right]\right\} & =1+\sum_{n=1}^{\infty} s^{n} \mathbf{E}\left[e^{\lambda S_{n}} ; \Gamma^{\prime}>n\right] \\
& =\int_{0}^{+\infty} e^{-\lambda x} U_{s}(d x),
\end{aligned}
$$

where

$$
\Gamma^{\prime}:=\min \left(n \in \mathbb{N}, S_{n} \geq 0\right)
$$

and

$$
U_{s}(x)=\sum_{n=0}^{\infty} s^{n} \mathbf{P}\left(S_{n} \geq-x ; \Gamma^{\prime}>n\right), x \geq 0 .
$$

Therefore,

$$
\begin{aligned}
\sum_{n=0}^{\infty} s^{n} \mathbf{P}\left(L_{n} \geq-x\right) & =U_{s}(x) \exp \left\{\sum_{n=1}^{\infty} \frac{s^{n}}{n} \mathbf{P}\left(S_{n} \geq 0\right)\right\} \\
& =U_{s}(x) \sum_{n=1}^{\infty} s^{n} \mathbf{P}\left(L_{n} \geq 0\right)
\end{aligned}
$$

for $x \geq 0$. Note that by the duality principle for random walks (see, for instance, Kersting and Vatutin (2017) p. 63),

$$
\begin{aligned}
\lim _{s \uparrow 1} U_{s}(x) & =\sum_{n=0}^{\infty} \mathbf{P}\left(S_{n} \geq-x ; \Gamma^{\prime}>n\right) \\
& =1+\sum_{n=1}^{\infty} \mathbf{P}\left(S_{n} \geq-x ; S_{i}<0, i=1, \ldots, n\right) \\
& =1+\sum_{n=1}^{\infty} \mathbf{P}\left(S_{n} \geq-x ; S_{n}<S_{j}, j=0,1, \ldots, n-1\right) \\
& =1+\sum_{n=1}^{\infty} \sum_{r=1}^{n} \mathbf{P}\left(S_{n} \geq-x ; \gamma_{r}=n\right) \\
& =1+\sum_{r=1}^{\infty} \sum_{n=r}^{\infty} \mathbf{P}\left(S_{n} \geq-x ; \gamma_{r}=n\right)=1+\sum_{r=1}^{\infty} \mathbf{P}\left(S_{\gamma_{r}} \geq-x\right)=U(x)
\end{aligned}
$$

On the other hand, if $s \uparrow 1$ then (2.1) and an application of Corollary 1.7.3 in Bingham et al. (1989) with $\rho=0$ give for $p>q$,

$$
\sum_{n=0}^{\infty} s^{n} \mathbf{P}\left(L_{n} \geq 0\right) \sim \sum_{n=0}^{\infty} s^{n} \frac{\mathfrak{l}_{2}(n)}{n} \sim \hat{\mathfrak{l}}_{2}\left(\frac{1}{1-s}\right)
$$

while (2.2) and again an application of Corollary 1.7.3 in Bingham et al. (1989) but now with $\rho=1$ justify, for $p<q$ the asymptotics

$$
\sum_{n=0}^{\infty} s^{n} \mathbf{P}\left(L_{n} \geq 0\right) \sim \sum_{n=0}^{\infty} s^{n} \mathfrak{l}_{3}(n) \sim \frac{\mathfrak{l}_{3}(1 /(1-s))}{1-s} .
$$


Thus if $p>q$ then, as $s \uparrow 1$

$$
\sum_{n=0}^{\infty} s^{n} \mathbf{P}\left(L_{n} \geq-x\right) \sim U(x) \hat{\mathfrak{l}}_{2}\left(\frac{1}{1-s}\right),
$$

and if $p<q$ then, as $s \uparrow 1$

$$
\sum_{n=0}^{\infty} s^{n} \mathbf{P}\left(L_{n} \geq-x\right) \sim U(x) \frac{\mathfrak{l}_{3}(1 /(1-s))}{1-s} .
$$

Using (2.6), (2.8) and the monotonicity of $U_{s}(x)$ in $s$ we get, for $p>q$

$$
\sum_{n=0}^{\infty} s^{n} \mathbf{P}\left(L_{n} \geq-x\right) \leq U(x) \sum_{n=0}^{\infty} s^{n} \mathbf{P}\left(L_{n} \geq 0\right) \sim U(x) \hat{\mathfrak{l}}_{2}\left(\frac{1}{1-s}\right) .
$$

Since $\mathbf{P}\left(L_{n} \geq-x\right)$ is nonincreasing with $n$, we have for $p>q$

$$
\begin{aligned}
\frac{n}{2}\left(1-\frac{1}{n}\right)^{n} \mathbf{P}\left(L_{n} \geq-x\right) & \leq \sum_{n / 2 \leq m \leq n}\left(1-\frac{1}{n}\right)^{m} \mathbf{P}\left(L_{m} \geq-x\right) \\
& \leq C U(x) \hat{\mathfrak{l}}_{2}(n),
\end{aligned}
$$

and, similarly, for $p<q$

$$
\frac{n}{2}\left(1-\frac{1}{n}\right)^{n} \mathbf{P}\left(L_{n} \geq-x\right) \leq C U(x) n \mathfrak{l}_{3}(n) .
$$

As a result

$$
\mathbf{P}\left(L_{n} \geq-x\right) \leq\left\{\begin{array}{cl}
C U(x) n^{-1} \hat{\mathfrak{l}}_{2}(n) & \text { if } \quad p>q, \\
C U(x) \mathfrak{l}_{3}(n) & \text { if } \quad p<q .
\end{array}\right.
$$

By the same arguments and (2.3) we have as $s \uparrow 1$

$$
\sum_{n=1}^{\infty} s^{n} \mathbf{P}\left(M_{n}<0\right) \sim \sum_{n=1}^{\infty} s^{n} \mathfrak{l}_{4}(n) \sim \frac{\mathfrak{l}_{4}(1 /(1-s))}{1-s}
$$

for $p>q$, and by (2.4)

$$
\sum_{n=1}^{\infty} s^{n} \mathbf{P}\left(M_{n}<0\right) \sim \sum_{n=1}^{\infty} s^{n} \frac{\mathfrak{l}_{5}(n)}{n} \sim \hat{\mathfrak{l}}_{5}\left(\frac{1}{1-s}\right)
$$

for $p<q$. Thus

$$
\mathbf{P}\left(M_{n}<x\right) \leq\left\{\begin{array}{cl}
C V(x) \mathfrak{l}_{4}(n) & \text { if } \quad p>q, \\
C V(x) n^{-1} \hat{\mathfrak{l}}_{5}(n) & \text { if } \quad p<q .
\end{array}\right.
$$

This ends the proof.

Remark 2.3. Observe that

$$
\begin{aligned}
\sum_{n=1}^{\infty} s^{n} \mathbf{P}\left(L_{n}\right. & \geq 0) \sum_{n=1}^{\infty} s^{n} \mathbf{P}\left(M_{n}<0\right) \\
& =\exp \left\{\sum_{n=1}^{\infty} \frac{s^{n}}{n} \mathbf{P}\left(S_{n} \geq 0\right)\right\} \times \exp \left\{\sum_{n=1}^{\infty} \frac{s^{n}}{n} \mathbf{P}\left(S_{n}<0\right)\right\}=\frac{1}{1-s} .
\end{aligned}
$$


Thus, as $n \rightarrow \infty$

$$
\hat{\mathfrak{l}}_{2}(n) \mathfrak{l}_{4}(n) \sim 1, \hat{\mathfrak{l}}_{5}(n) \mathfrak{l}_{3}(n) \sim 1
$$

Set

$$
b_{n}:=\left(n a_{n}\right)^{-1}, \quad n \in \mathbb{N}
$$

The next statement describes some properties of the running extrema of $\mathcal{S}$.

Lemma 2.4. (compare with Proposition 2.3 in Afanasyev et al. (2012)) Assume that Conditions (1.4) and (1.8) hold. Then there exists a constant $c$ such that, uniformly for all $x, y \geq 0$ and all $n \in \mathbb{N}$

$$
\mathbf{P}_{x}\left(L_{n} \geq 0, y-1 \leq S_{n}<y\right) \leq c b_{n} U(x) V(y)
$$

and

$$
\mathbf{P}_{-x}\left(M_{n}<0,-y \leq S_{n}<-y+1\right) \leq c b_{n} V(x) U(y) .
$$

Proof: We prove the latter statement only. Since the density of any $\alpha$-stable law is bounded, it follows from Gnedenko and Kolmogorov (1954) and Stone (1965) local limit theorems that there exists a finite constant $C$ such that for all $n \in \mathbb{N}$ and all $z, \Delta \geq 0$

$$
\mathbf{P}\left(S_{n} \in[-z,-z+\Delta)\right) \leq \frac{C \Delta}{a_{n}} .
$$

Let $x, y \geq 0, \mathcal{S}^{\prime}$ be the dual random walk

$$
S_{i}^{\prime}=S_{n}-S_{n-i}
$$

and $L_{i}^{\prime}, i \leq n$, the corresponding minima. Denote

$$
\begin{aligned}
A_{n} & :=\left\{M_{\lfloor n / 3\rfloor}<x\right\} \\
A_{n}^{\prime} & :=\left\{L_{\lfloor n / 3\rfloor}^{\prime} \geq-y\right\} \\
A_{n}^{\prime \prime} & :=\left\{x-y \leq S_{n}<x-y+1\right\} \\
& =\left\{x-y-T_{n} \leq S_{\lfloor 2 n / 3\rfloor}-S_{\lfloor n / 3\rfloor}<x-y-T_{n}+1\right\},
\end{aligned}
$$

with

$$
T_{n}:=S_{\lfloor n / 3\rfloor}+S_{n}-S_{\lfloor 2 n / 3\rfloor} .
$$

Let $\mathcal{A}_{n}$ be the $\sigma$-field generated by $X_{1}, \ldots, X_{\lfloor n / 3\rfloor}$ and $X_{\lfloor 2 n / 3\rfloor+1}, \ldots, X_{n}$. Then $T_{n}$ is $\mathcal{A}_{n}$-measurable, whereas $S_{\lfloor 2 n / 3\rfloor}-S_{\lfloor n / 3\rfloor}$ is independent of $\mathcal{A}_{n}$. Consequently from (2.11) and the fact that $\left\{a_{n}, n \in \mathbb{N}\right\}$ is regularly varying there is a $c>0$ such that

$$
\mathbf{P}\left(A_{n}^{\prime \prime} \mid \mathcal{A}_{n}\right) \leq c a_{n}^{-1} .
$$

Since $A_{n}, A_{n}^{\prime}$ are $\mathcal{A}_{n}$-measurable and independent, it follows that

$$
\mathbf{P}\left(A_{n} \cap A_{n}^{\prime} \cap A_{n}^{\prime \prime}\right) \leq c a_{n}^{-1} \mathbf{P}\left(A_{n}\right) \mathbf{P}\left(A_{n}^{\prime}\right) .
$$

Moreover, according to Lemma 2.2

$$
\mathbf{P}\left(L_{\lfloor n / 3\rfloor}^{\prime} \geq-y\right) \leq c_{1} U(y) n^{-1} \hat{\mathfrak{l}}_{2}(n), \quad \mathbf{P}\left(M_{\lfloor n / 3\rfloor}<x\right) \leq c_{2} V(x) \mathfrak{l}_{4}(n),
$$

if $p>q$ and

$$
\mathbf{P}\left(L_{\lfloor n / 3\rfloor}^{\prime} \geq-y\right) \leq c_{1} U(y) \mathfrak{l}_{3}(n), \quad \mathbf{P}\left(M_{\lfloor n / 3\rfloor}<x\right) \leq c_{2} V(x) n^{-1} \hat{\mathfrak{l}}_{5}(n),
$$

if $p<q$. This and (2.9) give the uniform estimate

$$
\mathbf{P}\left(A_{n} \cap A_{n}^{\prime} \cap A_{n}^{\prime \prime}\right) \leq c V(x) U(y) b_{n}
$$


for $c$ sufficiently large. Now notice that

$$
\left\{M_{n}<x, x-y \leq S_{n}<x-y+1\right\} \subset A_{n} \cap A_{n}^{\prime} \cap A_{n}^{\prime \prime} .
$$

The fact that the event on the left hand side is included in $A_{n} \cap A_{n}^{\prime \prime}$ is straightforward. It is also included in $A_{n}^{\prime}$ due to the following series of inequalities, which hold for any $0 \leq i \leq n$ on the event $\left\{x-y \leq S_{n}, M_{n}<x\right\}$ :

$$
x-y \leq S_{n}-S_{i}+S_{i} \leq S_{n}-S_{i}+M_{n} \leq S_{n}-S_{i}+x=S_{n-i}^{\prime}+x .
$$

This ends the proof.

We have now all the tools needed to prove the following statement.

Lemma 2.5. Assume that Conditions (1.4) and (1.8) hold. Then for every $x \geq 0$ as $n \rightarrow \infty$

1) if $p>q$ then

$$
\begin{aligned}
\mathbf{P}\left(L_{n} \geq-x\right) & \sim U(x) \mathbf{P}\left(L_{n} \geq 0\right) \sim U(x) \frac{\mathfrak{l}_{2}(n)}{n}, \\
\mathbf{P}\left(M_{n}<x\right) & \sim V(x) \mathbf{P}\left(M_{n}<0\right) \sim V(x) \mathfrak{l}_{4}(n) ;
\end{aligned}
$$

2) if $p<q$ then

$$
\begin{aligned}
\mathbf{P}\left(L_{n} \geq-x\right) & \sim U(x) \mathbf{P}\left(L_{n} \geq 0\right) \sim U(x) \mathfrak{l}_{3}(n), \\
\mathbf{P}\left(M_{n}<x\right) & \sim V(x) \mathbf{P}\left(M_{n}<0\right) \sim V(x) \frac{\mathfrak{l}_{5}(n)}{n} .
\end{aligned}
$$

Proof: As the derivations of the four equivalents are similar, we only check the first one. Let

$$
\tau_{n}:=\min \left\{j \leq n: S_{j}=L_{n}\right\} .
$$

We have

$$
\begin{aligned}
\mathbf{P}\left(L_{n} \geq-x\right) & =\sum_{j=0}^{n} \mathbf{P}\left(L_{n-j} \geq 0\right) \mathbf{P}\left(S_{j} \geq-x ; \tau_{j}=j\right) \\
& =\sum_{j=0}^{n} \mathbf{P}\left(L_{n-j} \geq 0\right) \mathbf{P}\left(S_{j} \geq-x ; M_{j}<0\right),
\end{aligned}
$$

where we used the duality principle as in (2.7). In view of $(2.1)$, for any $\varepsilon \in(0,1)$ and $j \leq \varepsilon n$,

$$
\mathbf{P}\left(L_{n-j} \geq 0\right) \sim \frac{n}{n-j} \mathbf{P}\left(L_{n} \geq 0\right), \quad n \rightarrow \infty .
$$

Moreover, from (2.7), we have

$$
\sum_{j=0}^{n \varepsilon} \mathbf{P}\left(S_{j} \geq-x ; M_{j}<0\right)=\sum_{j=0}^{n \varepsilon} \mathbf{P}\left(S_{j} \geq-x ; \Gamma^{\prime}>j\right) \sim U(x), \quad n \rightarrow \infty .
$$

We deduce that

$$
\sum_{j=0}^{n \varepsilon} \mathbf{P}\left(L_{n-j} \geq 0\right) \mathbf{P}\left(S_{j} \geq-x ; M_{j}<0\right)-\mathbf{P}\left(L_{n} \geq 0\right) U(x)=O(\varepsilon) \mathbf{P}\left(L_{n} \geq 0\right) U(x)
$$


when $n$ is large enough. Further, by (2.10), (2.1) and (1.6), for any $\delta>0$

$$
\begin{aligned}
\sum_{j=n \varepsilon}^{n} \mathbf{P}\left(L_{n-j} \geq 0\right) \mathbf{P}\left(S_{j} \geq-x ; M_{j}<0\right) & \leq C b_{n} x U(x) \sum_{j=n \varepsilon}^{n} \mathbf{P}\left(L_{n-j} \geq 0\right) \\
& \leq \frac{C x U(x)}{n a_{n}} \hat{\mathfrak{l}}_{2}(n) \\
& =\frac{C x U(x)}{n^{2}} \frac{\hat{\mathfrak{l}}_{2}(n)}{\mathfrak{l}_{1}(n)}=o\left(\frac{1}{n^{2-\delta}}\right) \\
& =o\left(\mathbf{P}\left(L_{n} \geq 0\right)\right), \quad n \rightarrow \infty,(2 .
\end{aligned}
$$

since $\left(\hat{\mathfrak{l}}_{2}(n) / \mathfrak{l}_{1}(n)\right) n^{-\delta} \rightarrow 0$ as $n \rightarrow \infty$ for any $\delta>0$. Combining (2.15) and (2.16) and letting $\varepsilon \rightarrow 0$ give (2.12).

The last result of this section is a technical statement which will be needed in the proof of Theorem 1.6. As Lemma 2.5, it is a consequence of Lemma 2.4 and can be proven in the same way as Corollary 2.4 in Afanasyev et al. (2012).

Lemma 2.6. Assume that Conditions (1.4) and (1.8) hold. For any $\theta>0$ there exists a finite $c$ (depending on $\theta$ ) such that for all $x, y \geq 0$

$$
\mathbf{E}_{x}\left[e^{-\theta S_{n}} ; L_{n} \geq 0, S_{n} \geq y\right] \leq c b_{n} V(x) U(y) e^{-\theta y}
$$

and

$$
\mathbf{E}_{-x}\left[e^{\theta S_{n}} ; M_{n}<0, S_{n}<-y\right] \leq c b_{n} V(y) U(x) e^{\theta y} .
$$

\section{Change of measure}

Recall the definition of the renewal function $U$ in (1.12). One of its fundamental properties is the identity (see, for instance, Kozlov, 1976; Bertoin and Doney, 1994)

$$
\mathbf{E}[U(x+X) ; X+x \geq 0]=U(x), x \geq 0 .
$$

This property has often been used to construct a change of probability measure (see for instance Geiger and Kersting, 2000), and we will use such a construction in our proof.

Denote by $\mathcal{F}$ the filtration consisting of the $\sigma$-algebras $\mathcal{F}_{n}$ generated by the random variables $S_{0}, \ldots, S_{n}$ and $Z_{0}, \ldots, Z_{n}$. Taking into account $U(0)=1$ we may introduce probability measures $\mathbf{P}_{n}^{+}$on the $\sigma$-fields $\mathcal{F}_{n}$ by means of the densities

$$
d \mathbf{P}_{n}^{+}:=U\left(S_{n}\right) \mathbf{1}_{\left\{L_{n} \geq 0\right\}} d \mathbf{P} .
$$

Because of the martingale property the measures are consistent, i.e., $\mathbf{P}_{n+1}^{+} \mid \mathcal{F}_{n}=$ $\mathbf{P}_{n}^{+}$. Therefore (choosing a suitable underlying probability space), there exists a probability measure $\mathbf{P}^{+}$on the $\sigma$-field $\mathcal{F}_{\infty}:=\bigvee_{n} \mathcal{F}_{n}$ such that

$$
\mathbf{P}^{+} \mid \mathcal{F}_{n}=\mathbf{P}_{n}^{+}, \quad n \geq 0 .
$$

We note that (3.1) can be rewritten as

$$
\mathbf{E}^{+}\left[Y_{n}\right]=\mathbf{E}\left[Y_{n} U\left(S_{n}\right) ; L_{n} \geq 0\right]
$$

for every $\mathcal{F}_{n}$-measurable nonnegative random variable $Y_{n}$. This change of measure is the well-known Doob $h$-transform from the theory of Markov processes. In particular, under $\mathbf{P}^{+}$the process $S$ becomes a Markov chain with state space $\mathbb{R}_{0}^{+}$and 
transition kernel

$$
P^{+}(x ; d y):=\frac{1}{U(x)} \mathbf{P}(x+X \in d y) U(y), \quad x \geq 0 .
$$

In our context, we can show that $\mathbf{P}^{+}$can be realised as the limit of the probability of the process conditioned to live in a nonnegative environment (in the sense that the running infimum is null). It is the content of the next lemma, and will allow us to link the survival probability of the population process to the probability for the running infimum to be null, in order to prove Theorem 1.6.

Lemma 3.1. (compare with Lemma 2.5 in Afanasyev et al., 2005) Assume that Conditions (1.4) and (1.8) hold. For $k \in \mathbb{N}$ let $Y_{k}$ be a bounded real-valued $\mathcal{F}_{k-}$ measurable random variable. Then, as $n \rightarrow \infty$,

$$
\mathbf{E}\left[Y_{k} \mid L_{n} \geq 0\right] \rightarrow \mathbf{E}^{+} Y_{k}
$$

More generally, let $Y_{1}, Y_{2}, \ldots$ be a uniformly bounded sequence of real-valued random variables adapted to the filtration $\mathcal{F}$, which converges $\mathbf{P}^{+}$-a.s. to some random variable $Y_{\infty}$. Then, as $n \rightarrow \infty$,

$$
\mathbf{E}\left[Y_{n} \mid L_{n} \geq 0\right] \rightarrow \mathbf{E}^{+} Y_{\infty}
$$

Proof: The proof of this lemma in the case $p>q$ coincides with the proof of Lemma 2.5 in Afanasyev et al. (2005) when taking $\rho=0$ and we omit it. In the case $p<q$ some modifications are needed to check the second claim of the lemma. Namely, writing

$$
m_{l}(x):=\mathbf{P}\left(L_{l} \geq-x\right) \text { for } \quad x \geq 0, l \in \mathbb{N}
$$

and using (2.5), (2.13) and (3.2) we deduce for $\lambda>1, k \leq n$ and $n$ large enough, the existence of a finite $C$ such that

$$
\begin{aligned}
\left|\mathbf{E}\left[Y_{n}-Y_{k} \mid L_{\lfloor\lambda n\rfloor} \geq 0\right]\right| & =\left|\mathbf{E}\left[\left(Y_{n}-Y_{k}\right) \frac{\mathbf{1}_{\left\{\inf _{0 \leq k \leq\lfloor(\lambda-1) n\rfloor} S_{n+k}-S_{n} \geq-S_{n}\right\}}}{m_{\lfloor\lambda n\rfloor}(0)} \mathbf{1}_{\left\{L_{n} \geq 0\right\}}\right]\right| \\
& \leq \mathbf{E}\left[\left|Y_{n}-Y_{k}\right| \frac{m_{\lfloor(\lambda-1) n\rfloor}\left(S_{n}\right)}{m_{\lfloor\lambda n\rfloor}(0)} \mathbf{1}_{\left\{L_{n} \geq 0\right\}}\right] \\
& \leq C \mathbf{E}\left[\left|Y_{n}-Y_{k}\right| U\left(S_{n}\right) \mathbf{1}_{\left\{L_{n} \geq 0\right\}}\right] \\
& =C \mathbf{E}^{+}\left[\left|Y_{n}-Y_{k}\right|\right],
\end{aligned}
$$

where we have used that for any $k \geq 0, S_{n+k}-S_{n}$ is independent of $\mathcal{F}_{n}$. Letting sequentially $n$ and $k$ go to infinity and applying the dominated convergence theorem, we obtain that the right hand side of the previous series of inequalities vanishes. Applying now the first claim of the lemma and using the fact that $n \mapsto \mathbf{P}\left(L_{n} \geq 0\right)$ is slowly varying we obtain

$$
\mathbf{E}\left[Y_{n} ; L_{\lfloor\lambda n\rfloor} \geq 0\right]=\left(\mathbf{E}^{+}\left[Y_{\infty}\right]+o(1)\right) \mathbf{P}\left(L_{\lfloor\lambda n\rfloor} \geq 0\right)=\left(\mathbf{E}^{+}\left[Y_{\infty}\right]+o(1)\right) \mathbf{P}\left(L_{n} \geq 0\right)
$$

and

$$
\mathbf{E}\left[Y_{n} ; L_{n} \geq 0\right]-\mathbf{E}\left[Y_{n} ; L_{\lfloor\lambda n\rfloor} \geq 0\right]=o\left(\mathbf{P}\left(L_{n} \geq 0\right)\right) .
$$

This ends the proof.

Let $\nu \geq 1$ be the time of the first prospective minimal value of $\mathcal{S}$, i.e., a minimal value with respect to the future development of the walk,

$$
\nu:=\min \left\{m \in \mathbb{N}: S_{m+i} \geq S_{m} \text { for all } i \geq 0\right\} .
$$


Moreover, let $\iota \in \mathbb{N}$ be the first weak ascending ladder epoch of $S$,

$$
\iota:=\min \left\{m \in \mathbb{N}: S_{m} \geq 0\right\} .
$$

We denote

$$
\widetilde{f}_{n}:=f_{\nu+n} \text { and } \widetilde{S}_{n}:=S_{\nu+n}-S_{\nu}, \quad n \in \mathbb{N} .
$$

The previous result allows us to rigorously express what we mean by living in a good environment for the population process. The next lemma and its proof are the same as Lemma 2.6 in Afanasyev et al. (2005) and its proof. We thus do not provide it and refer the reader to Afanasyev et al. (2005).

Lemma 3.2. (see Lemma 2.6 in Afanasyev et al., 2005) Suppose that $\iota<\infty \mathbf{P}$-a.s. Then $\nu<\infty \mathbf{P}^{+}$-a.s. and

(1) $\left(f_{1}, f_{2}, \ldots\right)$ and $\left(\widetilde{f}_{1}, \widetilde{f}_{2}, \ldots\right)$ are identically distributed with respect to $\mathbf{P}^{+}$;

(2) $\left(\nu, f_{1}, \ldots, f_{\nu}\right)$ and $\left(\widetilde{f}_{1}, \widetilde{f}_{2}, \ldots\right)$ are independent with respect to $\mathbf{P}^{+}$;

(3) $\mathbf{P}^{+}\left\{\nu=k, S_{\nu} \in d x\right\}=\mathbf{P}\left\{\iota=k, S_{\iota} \in d x\right\}$ for all $k \geq 1$.

\section{Proof of Theorem 1.6}

Thanks to the results we have collected in the previous sections, we are now able to prove our first main result. We have already demonstrated (Lemmas 3.1 and 3.2) that we can divide the survival probability of $\mathcal{Z}$ until time $n$ into two parts: the probability for the process to survive until the time when the running infimum $L_{n}$ is attained for the first time, and the probability that the process $\mathcal{Z}$ survives in a "good" environment, i.e., in an environment with a running infimum of $L$ null. We still have to prove that the population indeed has a nonnegligible probability to survive in this good environment, for large $n$. It is the content of the next result.

Let

$$
\eta_{k}:=\sum_{y=0}^{\infty} y(y-1) f_{k}[y] /\left(\sum_{y=0}^{\infty} y f_{k}[y]\right)^{2}, \quad k \in \mathbb{N} .
$$

Lemma 4.1. Assume that Conditions (1.4) and (1.8) hold. If $p>q$, and Conditions $\boldsymbol{A}$ and $\boldsymbol{C}$ hold or if $p<q$ and Condition $\boldsymbol{B}$ holds, then

$$
\sum_{k=0}^{\infty} \eta_{k+1} e^{-S_{k}}<\infty \quad \mathbf{P}^{+} \text {-a.s. }
$$

Proof: Let us first assume that $p>q$, and Conditions A and C hold. Recall the definition of the standardized truncated second moment of the environment in (1.10). Following Afanasyev et al. (2005) Equation (2.24) we have the following bound, for any $a \in \mathbb{N}$,

$$
\sum_{k=0}^{\infty} \eta_{k+1} e^{-S_{k}} \leq a \sum_{k=0}^{\infty} e^{-S_{k}}+\sum_{k=0}^{\infty} \zeta_{k+1}(a) e^{-S_{k}}=: \mathcal{A}_{a}+\mathcal{B}_{a} .
$$

The first step of the proof consists in bounding the two sums by using the times $0:=\nu(0)<\nu(1)<\cdots$ of prospective minima of $\mathcal{S}$, defined by

$$
\nu(j):=\min \left\{m>\nu(j-1): S_{m+i} \geq S_{m} \text { for all } i \geq 0\right\}, \quad j \in \mathbb{N} .
$$

By definition,

$$
S_{k} \geq S_{\nu(j)}, \text { if } k \geq \nu(j)
$$


Thus, we get

$$
\mathcal{A}_{a} \leq a \sum_{j=0}^{\infty}(\nu(j+1)-\nu(j)) e^{-S_{\nu(j)}}
$$

and

$$
\mathcal{B}_{a} \leq \sum_{j=0}^{\infty}\left(\sum_{k=\nu(j)+1}^{\nu(j+1)} \zeta_{k}(a)\right) e^{-S_{\nu(j)}}
$$

Now we aim at bounding the variables $\nu(j)$. For the sake of readability, let us introduce

$$
\nu_{j}=\nu(j)-\nu(j-1), \quad j \in \mathbb{N} .
$$

By Lemma 3.2.(1) and (2), $\nu(j)$ is the sum of $j$ nonnegative i.i.d. random variables, each having the distribution of $\nu=\nu(1)=\nu_{1}$. Lemma 3.2.(3) and (2.3) imply for large $k$

$$
\begin{aligned}
\mathbf{P}^{+}(\nu>k) & =\mathbf{P}\{\iota>k\}=\mathbf{P}\left\{M_{k}<0\right\} \\
& \leq 2 \mathfrak{l}_{4}(k)=2 / \Lambda(k) .
\end{aligned}
$$

These estimates and Condition $\mathbf{C}$ imply

$$
\sum_{j=1}^{\infty} \mathbf{P}^{+}\left(\nu_{j}>g(j)\right) \leq 2 \sum_{j=1}^{\infty} 1 / \Lambda(g(j))<\infty .
$$

Hence, by the Borel-Cantelli lemma there will be $\mathbf{P}^{+}-a . s$. only a finite number cases when $\nu_{j}>g(j)$. And as $g(i)=e^{o(i)}, i \rightarrow \infty$, for any $\gamma>0$,

$$
\sum_{i=0}^{j} g(i)=o\left(e^{\gamma j}\right), \quad j \rightarrow \infty .
$$

Thus, there will be $\mathbf{P}^{+}-$a.s. only a finite number of cases when $\nu(j)>e^{\gamma j}$.

Now we would like to bound the term

$$
\sum_{k=\nu(j)+1}^{\nu(j+1)} \zeta_{k}(a)
$$

in order to show that the random variable $\mathcal{B}_{a}$ is almost surely finite. The first step to obtain this bound is to use the inequality (2.25) in Afanasyev et al. (2005), that we now recall: for any $x \geq 0$,

$$
\mathbf{P}^{+}\left(\zeta_{k}(a)>x\right) \leq \mathbf{P}\left(\zeta_{1}(a)>x\right)+\mathbf{E}\left[U\left(X_{1}\right) ; \zeta_{1}(a)>x\right] \mathbf{P}\left(L_{k-1} \geq 0\right) .
$$

Applying it with $x=k^{\alpha / \gamma}$ (with $\alpha>0$ to be selected later on) and using the Markov inequality as well as Condition $\mathbf{A}$ yields for any $k \in \mathbb{N}$,

$$
\mathbf{P}^{+}\left(\zeta_{k}(a)>k^{\alpha / \gamma}\right) \leq \frac{c}{k^{\alpha \beta / \gamma}}+\frac{c}{k^{\alpha \beta / \gamma}} \mathbf{P}\left(L_{k-1} \geq 0\right) \leq \frac{c}{k^{\alpha \beta / \gamma}}+\frac{c}{k^{\alpha \beta / \gamma}} \frac{\hat{\mathfrak{l}}_{2}(k)}{k}
$$

where we applied (2.5) and the value of $c$ can change from line to line. The constants $\alpha$ and $\beta$ are fixed. However, we know that $\gamma$ can be chosen as small as we want. In particular, we may select it in such a way that $\alpha \beta / \gamma=2$. Applying again the Borel-Cantelli lemma we deduce that there is $\mathbf{P}^{+}-$a.s. only a finite number of cases when $\zeta_{k}(a)>k^{\alpha / \gamma}$. 
Combining this fact with the previous results we obtain that for $j$ large enough and $k \in[\nu(j-1)+1, \nu(j)], \mathbf{P}^{+}-a . s .$,

$$
\zeta_{k}(a) \leq k^{\alpha / \gamma} \leq\left(e^{\gamma j}\right)^{\alpha / \gamma}=e^{\alpha j} \quad \text { and } \quad \nu_{j} \leq g(j) .
$$

Hence for $j$ large enough, $\mathbf{P}^{+}-$a.s.,

$$
\sum_{k=\nu(j-1)+1}^{\nu(j)} \zeta_{k}(a) \leq e^{\alpha j} \nu_{j} \leq e^{\alpha j} g(j)
$$

The last part of the proof consists in estimating the $S_{\nu(j)}$ from below. According to Lemma 3.2 (1) and (2), the random variable $S_{\nu(j)}$ is the sum of $j$ non-negative i.i.d. random variables with positive mean. Thus, there exists a $\lambda>0$ such that

$$
S_{\nu(j)} \geq \lambda j \quad \text { eventually } \quad \mathbf{P}^{+}-\text {a.s. }
$$

Choosing $\alpha<\lambda$ in the previous inequalities, we obtain

$$
\begin{aligned}
\sum_{k=0}^{\infty} \eta_{k+1} e^{-S_{k}} \leq \mathcal{A}_{a}+\mathcal{B}_{a} & \leq c \sum_{j=0}^{\infty}\left(a+e^{\alpha(j+1)}\right) \nu_{j+1} e^{-S_{\nu(j)}} \\
& \leq c \sum_{j=0}^{\infty} e^{\alpha(j+1)} g(j+1) e^{-\lambda j}<\infty \quad \mathbf{P}^{+} \text {-a.s. }
\end{aligned}
$$

where the value of $c$ can change from line to line. It ends the proof for the case $p>q$.

The proof for the case $p<q$ is the same as the proof of Lemma 2.7 in Afanasyev et al. (2005). Indeed, even if the authors of the mentioned paper assume $\rho \in(0,1)$, their proof remains valid when we take $\rho=1$ as it is the case when $p<q$.

Introduce iterations of probability generating functions $f_{1}(),. f_{2}(),. \ldots$ by setting

$$
f_{k, n}(s):=f_{k+1}\left(f_{k+2}\left(\ldots\left(f_{n}(s)\right) \ldots\right)\right)
$$

for $0 \leq k \leq n-1,0 \leq s \leq 1$, and letting $f_{n, n}(s):=s$. By definition,

$$
\mathbf{P}\left(Z_{n}>0 \mid f_{k+1}, \ldots, f_{n} ; Z_{k}=1\right)=1-f_{k, n}(0)
$$

and we have (see, for instance, formula (3.4) in Afanasyev et al. (2005))

$$
1-f_{0, n}(0) \geq\left(e^{-S_{n}}+\sum_{k=1}^{n-1} \eta_{k+1} e^{-S_{k}}\right)^{-1}
$$

implying by Lemma 4.1

$$
1-f_{0, \infty}(0):=\lim _{n \rightarrow \infty}\left(1-f_{0, n}(0)\right) \geq\left(\sum_{k=1}^{\infty} \eta_{k+1} e^{-S_{k}}\right)^{-1}>0 \quad \mathbf{P}^{+}-\text {a.s. }
$$

We finally provide the proof of the main result of this section. 
Proof of Theorem 1.6: Let us begin with the case $p>q$. Recall the definition of $\tau_{n}$ in (2.14). We write

$$
\begin{aligned}
\mathbf{P}\left(Z_{n}>0\right)= & \sum_{k=0}^{n} \mathbf{P}\left(Z_{n}>0 ; \tau_{n}=k\right) \\
= & \sum_{k=0}^{N} \mathbf{E}\left[1-f_{0, n}(0) ; \tau_{n}=k\right]+\sum_{k=N+1}^{n \varepsilon} \mathbf{E}\left[1-f_{0, n}(0) ; \tau_{n}=k\right] \\
& +\sum_{k=n \varepsilon+1}^{n} \mathbf{E}\left[1-f_{0, n}(0) ; \tau_{n}=k\right]
\end{aligned}
$$

for some $N \in \mathbb{N}$ to be precised later on, and a small positive $\varepsilon$. Let us first bound the second term in the right hand side of (4.2)

$$
\begin{aligned}
\sum_{k=N+1}^{n \varepsilon} \mathbf{E}\left[1-f_{0, n}(0) ; \tau_{n}=k\right] & \leq \sum_{k=N+1}^{n \varepsilon} \mathbf{E}\left[1-f_{0, k}(0) ; \tau_{n}=k\right] \\
& =\sum_{k=N+1}^{n \varepsilon} \mathbf{E}\left[1-f_{0, k}(0) ; \tau_{k}=k\right] \mathbf{P}\left(L_{n-k} \geq 0\right) \\
& \leq \sum_{k=N+1}^{n \varepsilon} \mathbf{E}\left[e^{S_{k}} ; \tau_{k}=k\right] \mathbf{P}\left(L_{n-k} \geq 0\right)
\end{aligned}
$$

By the duality principle for random walks and Lemma 2.6, with $x=y=0$, we have

$$
\mathbf{E}\left[e^{S_{k}} ; \tau_{k}=k\right]=\mathbf{E}\left[e^{S_{k}} ; M_{k}<0\right] \leq c b_{k} .
$$

This estimate, the equivalence

$$
b_{k}=\frac{1}{k a_{k}} \sim \frac{1}{k^{2} \mathfrak{l}_{1}(k)}, \quad(k \rightarrow \infty)
$$

and (2.1) give

$$
\begin{aligned}
\sum_{k=N+1}^{n \varepsilon} \mathbf{E}\left[1-f_{0, n}(0) ; \tau_{n}=k\right] & \leq \mathbf{P}\left(L_{n(1-\varepsilon)} \geq 0\right) \sum_{k=N+1}^{\infty} \frac{1}{k^{2} \mathfrak{l}_{1}(k)} \\
& \leq C \frac{\mathbf{P}\left(L_{n(1-\varepsilon)} \geq 0\right)}{a_{N}},
\end{aligned}
$$

where $C$ is a finite constant. Now we focus on the third part of the right hand side of (4.2). Similarly as for the second part, we have the following series of inequalities, where the value of the finite constant $C$ may change from line to line and may depend on $\varepsilon$ :

$$
\begin{aligned}
\sum_{k=n \varepsilon+1}^{n} \mathbf{E}\left[1-f_{0, n}(0) ; \tau_{n}=k\right] & \leq C \sum_{k=n \varepsilon+1}^{n} \frac{1}{k^{2} \mathfrak{l}_{1}(k)} \mathbf{P}\left(L_{n-k} \geq 0\right) \\
& \leq C \frac{\hat{\mathfrak{l}}_{2}(n)}{n^{2} \mathfrak{l}_{1}(n)}=o\left(\frac{1}{n^{3 / 2}}\right) .
\end{aligned}
$$

Finally,

$$
\sum_{k=0}^{N} \mathbf{E}\left[1-f_{0, n}(0) ; \tau_{n}=k\right]=\sum_{k=0}^{N} \mathbf{E}\left[1-f_{k, n}^{Z_{k}}(0) ; \tau_{k}=k, L_{k, n} \geq 0\right]
$$


where

$$
L_{k, n}=\min _{k \leq j \leq n}\left(S_{j}-S_{k}\right)
$$

Recalling Lemma 3.1 and using the independency and homogeneity of the environmental components we conclude that for $k \leq N$,

$$
\begin{aligned}
\mathbf{E}\left[1-f_{k, n}^{Z_{k}}(0) ; \tau_{k}=\right. & \left.k, L_{k, n} \geq 0\right] \\
=\sum_{j=1}^{\infty} \mathbf{P}\left(Z_{k}\right. & \left.=j, \tau_{k}=k\right) \mathbf{P}\left(L_{n-k} \geq 0\right) \mathbf{E}\left[1-f_{0, n-k}^{j}(0) \mid L_{n-k} \geq 0\right] \\
& \sim \mathbf{P}\left(L_{n} \geq 0\right) \sum_{j=1}^{\infty} \mathbf{P}\left(Z_{k}=j, \tau_{k}=k\right) \mathbf{E}^{+}\left[1-f_{0, \infty}^{j}(0)\right]
\end{aligned}
$$

as $n \rightarrow \infty$. Note that by Lemma 4.1 and (4.1)

$$
\mathbf{E}^{+}\left[1-f_{0, \infty}^{j}(0)\right] \geq \mathbf{E}^{+}\left[1-f_{0, \infty}(0)\right] \geq \mathbf{E}^{+}\left[\left(\sum_{k=0}^{\infty} \eta_{k+1} e^{-S_{k}}\right)^{-1}\right]>0
$$

Thus, letting first $n$ to infinity, then $\varepsilon$ to zero and, finally, $N$ to infinity we prove (1.16), with

$$
K_{1}=\sum_{k=0}^{\infty} \sum_{j=1}^{\infty} \mathbf{P}\left(Z_{k}=j, \tau_{k}=k\right) \mathbf{E}^{+}\left[1-f_{0, \infty}^{j}(0)\right]
$$

If we introduce the event

$$
A_{u . s .}:=\left\{Z_{n}>0 \text { for all } n \geq 0\right\}
$$

we may rewrite $K_{1}$ as follows:

$$
K_{1}=\sum_{k=0}^{\infty} \mathbf{E}\left[\mathbf{P}_{Z_{k}}^{+}\left(A_{u . s .}\right), \tau_{k}=k\right] .
$$

The proof for the case $q>p$ is very similar. The only difference is when looking for an equivalent of Equation (4.3). Applying (2.2) yields

$$
\begin{aligned}
\sum_{k=n \varepsilon+1}^{n} \mathbf{E}\left[1-f_{0, n}(0) ; \tau_{n}=k\right] & \leq C \sum_{k=n \varepsilon+1}^{n} \frac{1}{k^{2} \mathfrak{l}_{1}(k)} \mathbf{P}\left(L_{n-k} \geq 0\right) \\
& \leq C_{\varepsilon} \frac{\mathfrak{l}_{3}(n)}{n \mathfrak{l}_{1}(n)}=o\left(\frac{1}{n^{1 / 2}}\right)=o\left(\mathbf{P}\left(L_{n} \geq 0\right)\right)
\end{aligned}
$$

We end the proof as for (1.16) and $K_{2}$ has the same expression as $K_{1}$.

\section{Proof of Theorem 1.7}

The proof of Theorem 1.7 is similar to the proof of Theorem 1.3 in Afanasyev et al. (2005). For this reason we mainly concentrate on the differences with the proof of the mentioned theorem assuming throughout that the assumptions of Theorem 1.7 are in force. 
Proof: First we note that the same as in Proposition 3.1 of Afanasyev et al. (2005) one can show that

$$
\mathbf{P}^{+}\left(Z_{n}>0 \text { for all } n \mid f_{1}, f_{2}, \ldots\right)>0 \quad \mathbf{P}^{+}-\text {a.s. }
$$

In particular,

$$
\mathbf{P}^{+}\left(Z_{n}>0 \text { for all } n\right)>0 \text {. }
$$

Moreover, as $n \rightarrow \infty$,

$$
e^{-S_{n}} Z_{n} \rightarrow W^{+} \quad \mathbf{P}^{+} \text {-a.s. },
$$

where the random variable $W^{+}$has the property

$$
\left\{W^{+}>0\right\}=\left\{Z_{n}>0 \text { for all } n\right\} \quad \mathbf{P}^{+} \text {-a.s. }
$$

Let $\phi$ be a bounded continuous function on the space $D[0,1]$ of càdlàg functions on the unit interval. For a fixed $s \in \mathbb{R}$ let $W^{s}$ denote the process with constant paths

$$
W_{t}^{s}:=e^{-s} W^{+}, \quad 0 \leq t \leq 1 .
$$

By (5.1) the process $e^{-s} X^{r_{n}, n}$ converges to $W^{s}$ as $n \geq r_{n} \rightarrow \infty$ in the Skorokhodmetric on the space $D[0,1] \mathbf{P}^{+}-a . s$.

$$
Y_{n}:=\phi\left(e^{-s} X^{r_{n}, n}\right) \mathbf{1}_{\left\{Z_{n}>0\right\}} \rightarrow Y_{\infty}:=\phi\left(W^{s}\right) \mathbf{1}_{\left\{W^{+}>0\right\}} \quad \mathbf{P}^{+} \text {-a.s. }
$$

For $r \leq n$ and $z \in \mathbb{N}_{0}$ define

$$
\psi(z, s, r, n):=\mathbf{E}_{z}\left[\phi\left(e^{-s} X^{r, n}\right) \mathbf{1}_{\left\{Z_{n}>0\right\}} ; L_{n} \geq 0\right] .
$$

Lemma 3.1 and (5.3) imply as $n \geq r_{n} \rightarrow \infty$

$$
\psi\left(z, s, r_{n}, n\right)=\mathbf{P}\left(L_{n} \geq 0\right)\left(\mathbf{E}_{z}^{+}\left[\phi\left(W^{s}\right) \mathbf{1}_{\left\{W^{+}>0\right\}}\right]+o(1)\right) .
$$

Now observe that for $k \leq r \leq n$

$$
\mathbf{E}\left[\phi\left(X^{r, n}\right) \mathbf{1}_{\left\{Z_{n}>0\right\}}, L_{k, n} \geq 0 \mid \mathcal{F}_{k}\right]=\psi\left(Z_{k}, S_{k}, r-k, n-k\right) \quad \mathbf{P}-a . s .
$$

Hence, using the arguments and conclusions of Theorem 1.6 we see that, for each fixed $N$

$$
\mathbf{E}\left[\phi\left(X^{r_{n}, n}\right) \mathbf{1}_{\left\{Z_{n}>0\right\}}\right]=\sum_{k=0}^{N} \mathbf{E}\left[\psi\left(Z_{k}, S_{k}, r_{n}-k, n-k\right) ; \tau_{k}=k\right]+\varepsilon_{N, n} \mathbf{P}\left(L_{n} \geq 0\right)
$$

where, for each fixed $k$

$$
\begin{aligned}
\lim _{n \geq r_{n} \rightarrow \infty} & \frac{\mathbf{E}\left[\psi\left(Z_{k}, S_{k}, r_{n}-k, n-k\right) ; \tau_{k}=k\right]}{\mathbf{P}\left(L_{n} \geq 0\right)} \\
= & \mathbf{E}\left[\mathbf{E}_{Z_{k}}^{+}\left[\phi\left(e^{-S_{k}} W^{+}\right) \mathbf{1}_{\left\{W^{+}>0\right\}}\right] ; Z_{k}>0, \tau_{k}=k\right]
\end{aligned}
$$

and

$$
\lim _{N \rightarrow \infty} \lim _{n \rightarrow \infty} \sup _{n, n}=0
$$

Thus,

$$
\begin{aligned}
\lim _{n \geq r_{n} \rightarrow \infty} \mathbf{E}\left[\phi\left(X^{r_{n}, n}\right) \mid Z_{n}>0\right] & =\frac{1}{K} \sum_{k=0}^{\infty} \mathbf{E}\left[\mathbf{E}_{Z_{k}}^{+}\left[\phi\left(e^{-S_{k}} W^{+}\right) \mathbf{1}_{\left\{W^{+}>0\right\}}\right] ; Z_{k}>0, \tau_{k}=k\right] \\
& =\int \phi(w) \lambda(d w)
\end{aligned}
$$


where (see Theorem 1.6) $K=K_{1}$ if $p>q, K=K_{2}$ if $p<q$ and

$$
\lambda(d w)=\frac{1}{K} \sum_{k=0}^{\infty} \mathbf{E}\left[\lambda_{Z_{k}, S_{k}}(d w) ; Z_{k}>0, \tau_{k}=k\right]
$$

with

$$
\lambda_{z, s}(d w)=\mathbf{P}_{z}^{+}\left(W^{s} \in d w, W^{+}>0\right) .
$$

Let $\phi \equiv 1$. By (4.5) and a similar expression for $K_{2}$ the limit in (5.5) is equal 1 in both cases. Thus, $\lambda$ is a probability measure. Besides, by (5.2) we see that $\lambda_{z, s}$ puts its entire mass on strictly positive constant functions and hence, so does $\lambda$. This completes the proof of Theorem 1.7.

\section{Acknowledgements}

The authors thank I. Kortchemski for attracting their attention to reference Kortchemski and Richier (2019) which allowed them to weaken the conditions for their main results to hold. C.S. thanks the CNRS for its financial support through its competitive funding programs on interdisciplinary research, the ANR ABIM 16CE40-0001 as well as the Chair "Modélisation Mathématique et Biodiversité" of VEOLIA-Ecole Polytechnique-MNHN-F.X. She also thanks the French Embassy in Russia for its financial support via the André Mazon program. The authors would like to thank two anonymous referees for their comments.

\section{References}

Afanasyev, V. I., Böinghoff, C., Kersting, G., and Vatutin, V. A. Limit theorems for weakly subcritical branching processes in random environment. J. Theoret. Probab., 25 (3), 703-732 (2012). MR2956209.

Afanasyev, V. I., Geiger, J., Kersting, G., and Vatutin, V. A. Criticality for branching processes in random environment. Ann. Probab., 33 (2), 645-673 (2005). MR2123206.

Allen, L. J. S. An introduction to stochastic processes with applications to biology. CRC Press, Boca Raton, FL, second edition (2011). ISBN 978-1-4398-1882-4. MR2560499.

Athreya, K. B. and Karlin, S. Branching processes with random environments. II. Limit theorems. Ann. Math. Statist., 42, 1843-1858 (1971a). MR298781.

Athreya, K. B. and Karlin, S. On branching processes with random environments. I. Extinction probabilities. Ann. Math. Statist., 42, 1499-1520 (1971b). MR298780.

Bansaye, V. and Böinghoff, C. Lower large deviations for supercritical branching processes in random environment. Tr. Mat. Inst. Steklova, 282 (Vetvyashchiesya Protsessy, Sluchaŭnye Bluzhdaniya, i Smezhnye Voprosy), 22-41 (2013). MR3308579.

Berger, Q. Notes on random walks in the Cauchy domain of attraction. Probab. Theory Related Fields, 175 (1-2), 1-44 (2019). MR4009704.

Bertoin, J. and Doney, R. A. On conditioning a random walk to stay nonnegative. Ann. Probab., 22 (4), 2152-2167 (1994). MR1331218.

Bertoin, J. and Doney, R. A. Spitzer's condition for random walks and Lévy processes. Ann. Inst. H. Poincaré Probab. Statist., 33 (2), 167-178 (1997). MR1443955. 
Bienaymé, I.-J. De la loi de multiplication et de la durée des familles. Soc. Philomat. Paris Extraits, Sér, 5 (37-39), 4 (1845).

Bingham, N. H., Goldie, C. M., and Teugels, J. L. Regular variation, volume 27 of Encyclopedia of Mathematics and its Applications. Cambridge University Press, Cambridge (1989). ISBN 0-521-37943-1. MR1015093.

Dyakonova, E. E., Geiger, J., and Vatutin, V. A. On the survival probability and a functional limit theorem for branching processes in random environment. Markov Process. Related Fields, 10 (2), 289-306 (2004). MR2082575.

Feller, W. An Introduction to Probability Theory and Its Applications. Vol. I. John Wiley \& Sons, Inc., New York, N.Y. (1950). MR0038583.

Geiger, J. and Kersting, G. The survival probability of a critical branching process in random environment. Teor. Veroyatnost. i Primenen., 45 (3), 607-615 (2000). MR1967796.

Geiger, J., Kersting, G., and Vatutin, V. A. Limit theorems for subcritical branching processes in random environment. Ann. Inst. H. Poincaré Probab. Statist., 39 (4), 593-620 (2003). MR1983172.

Gnedenko, B. V. and Kolmogorov, A. N. Limit distributions for sums of independent random variables. Addison-Wesley Publishing Company, Inc., Cambridge, Mass. (1954). MR0062975.

Haccou, P., Jagers, P., and Vatutin, V. A. Branching processes: variation, growth, and extinction of populations, volume 5 of Cambridge Studies in Adaptive Dynamics. Cambridge University Press, Cambridge; IIASA, Laxenburg (2007). ISBN 978-0-521-83220-5; 0-521-83220-9. MR2429372.

Kersting, G. and Vatutin, V. A. Discrete time branching processes in random environment. Wiley Online Library (2017). ISBN 978-1-786-30252-6.

Kortchemski, I. and Richier, L. Condensation in critical Cauchy Bienaymé-GaltonWatson trees. Ann. Appl. Probab., 29 (3), 1837-1877 (2019). MR3914558.

Kozlov, M. V. The asymptotic behavior of the probability of non-extinction of critical branching processes in a random environment. Teor. Verojatnost. i Primenen., 21 (4), 813-825 (1976). MR0428492.

Rogozin, B. A. Relatively stable walks. Teor. Verojatnost. i Primenen., 21 (2), 383-387 (1976). MR0423542.

Seneta, E. Regularly varying functions. Lecture Notes in Mathematics, Vol. 508. Springer-Verlag, Berlin-New York (1976). MR0453936.

Smith, W. L. and Wilkinson, W. E. On branching processes in random environments. Ann. Math. Statist., 40, 814-827 (1969). MR246380.

Stone, C. A local limit theorem for nonlattice multi-dimensional distribution functions. Ann. Math. Statist., 36, 546-551 (1965). MR175166.

Vatutin, V. and Dyakonova, E. Path to survival for the critical branching processes in a random environment. J. Appl. Probab., 54 (2), 588-602 (2017). MR3668485.

Vatutin, V. A. and D'yakonova, E. E. The initial stage of evolution of a weakly subcritical branching process in a random environment. Teor. Veroyatn. Primen., 64 (4), 671-691 (2019). MR4030816.

Vatutin, V. A., D'yakonova, E. E., and Sagitov, S. Evolution of branching processes in a random environment. Tr. Mat. Inst. Steklova, 282 (Vetvyashchiesya Protsessy, Sluchănye Bluzhdaniya, i Smezhnye Voprosy), 231-256 (2013). English version published in Proc. Steklov Inst. Math. 282 (2013), no. 1, 220-242. MR3308594. 
Watson, H. W. and Galton, F. On the probability of the extinction of families. The Journal of the Anthropological Institute of Great Britain and Ireland, 4, 138-144 (1875). 